Background Condoms decrease sexually transmitted infection(STI) transmission, good evidence supports this, if used correctly. This study investigates individuals understanding of the correct use of male latex condoms.

Methods Random individuals completed a questionnaire and were requested to conduct an observed demonstration of condom application, marked against criteria from British Association for Sexual Health and HIV(BASHH) guide for condom use and Centers for Disease Control and prevention(CDC) Condom fact sheet.

Results 127 responders, 45 participants in the observed demonstration(57\%males, 46\%females, age 12-66) 100\% believed they used condoms correctly, $68 \%$ were self taught. 100\% knew condoms were barrier contraception. $67 \%$ indicated condoms protection against all STIs and 5\% indicated no STI protection: $11 \%$ gonorrhoea and Chlamydia only, 10\% HSV and warts only and 7\% HIV only. $7 \%$ felt condoms decreased STI transmission during oral sex and 10\% during anal sex $(100 \%$ of men who have sex with men) During observed demonstration, 33\% correctly applied a condom. Mistakes: not squeezing air from condom, unrolling before applying and condom contact when opening. Factors stated to increase latex condom splitting: $25 \%$ penis size, $22 \%$ sexual vigour, $7 \%$ certain lubricants, $46 \%$ unsure $25 \%$ admitted to doubling condoms once since coitarche, all unaware of risk) $38 \%$ believed condoms not required throughout intercourse, $100 \%$ of these believed STI transmission was decreased if worn at the end.

Conclusion Perceived good condom technique, however, practical adherence to guidance is poor(particularly younger cohorts) Inadequate heterosexual awareness of STI transmission and prevention during oral and anal intercourse. Poor understanding of condom STI prevention, risks for condom splitting and timing of condom use. Good quality sexual education to include male condoms is important. Age of education is crucial capturing individuals before and timely to coitarche. Self teaching is common and requires quality accessible material. Opportunistic teaching is required, condom use competence should not be assumed.

\section{P3.361 MALE INVOLVEMENT IN THE VOICE MICROBICIDE TRIAL AT KAMPALA SITE UGANDA - A WORTHWHILE VENTURE}

doi:10.1136/sextrans-2013-051184.0814

P Ndawula, N Sophie, S Kabwigu, H Luwuge, A Zalwango, B Kamira, J Ggita. Makerere university-Johnhopkins university research collaboration, Kampala, Uganda

Background Makerere University-Johns Hopkins University research collaboration (MU-JHU) conducted a VOICE study which was a phase $2 \mathrm{~B}$ safety and effectiveness study of Tenofovir $1 \%$ gel, Tenofovir tablet and Truvada tablet for the prevention of HIV infection in women. The study encouraged women to disclose to their partners for support while using study products.

Methods Before and during recruitment, need for male involvement was emphasised. At screening and follow up visits, participants were informed about the importance of disclosing their participation to partners. Women were encouraged to invite their spouses to the clinic to know about the study and also receive HIV and syphilis testing. Men were invited verbally through their spouses; given reading materials to give to their partners before inviting them. Later, an invitation letter was developed inviting male partners to the clinic but given to those who opted for it. Male partners who came to the clinic were periodically invited for meetings to brainstorm issues about the study and general health.

Results Out of 322 participants, 140 accepted to take letters, 67 men turned up to the clinic, and 52 men tested for syphilis and HIV. 5 male partners' meetings were held and many concerns, which rotated around safety and effectiveness, were resolved, among which; whether it is safe for them to stop using a condom since their partners are on study products and whether the products will not affect their manhood.
Lessons learnt Male Partners who turned up to the clinic were very supportive in reminding their partners clinic appointments and product use for better adherence. Increased disclosure among discordant couples, and helped treating sexually transmitted infections.

Conclusion Male Involvement in microbicide studies is of utmost importance. Men need a proactive approach to get involved in clinic activities to support spouse in adherence and avoiding STD/STI reinfection.

\section{P3.362 MICROBICIDE USE DURING PREGNANCY: ACCEPTABILITY FOR PREVENTION/TREATMENT AND THE ROLE OF PARTNERS}

doi:10.1136/sextrans-2013-051184.0815

${ }^{1,2} \mathrm{M}$ Catallozzi, ${ }^{2} \mathrm{C}$ Y Williams, ${ }^{3} \mathrm{G} \mathrm{D}$ Zimet, ${ }^{4} \mathrm{~S}$ Gelber, ${ }^{2} \mathrm{~A}$ J Ratner, ${ }^{2} \mathbf{S}$ L Rosenthal. ${ }^{1}$ Columbia University Mailman School of Public Health, New York, NY, United States; ${ }^{2}$ Columbia University College of Physicians \& Surgeons, New York, NY, United States; ${ }^{3}$ Indiana University-Purdue University Indianapolis School of Medicine, Indianapolis, IN, United States; ${ }^{4}$ Weill Cornell Medical College, Cornell University, New York, NY, United States

Objectives To explore pregnant women's acceptance of microbicides for reproductive tract infections (RTIs).

Methods To date, we have analysed interview transcripts of 15 women in their third trimester who participated in a qualitative study on microbicide acceptability for treatment and prevention of bacterial vaginosis (BV) and role of partners in decision-making regarding product use. Interviews were coded for relevant themes. Women (mean age $=25.5$; range $=21-30$ ) were recruited from a clinic serving an inner-city minority population.

Results While few women reported BV history, most did not spontaneously provide specific BV knowledge. Most participants strongly endorsed treatment of any infection during pregnancy, however, responses to prevention ranged from ambivalence to strong support. Many women would make a decision based on perception of risk/ benefit to the baby. Other themes included: (1) side effects; (2) effectiveness; (3) perceived personal risk for RTI; (4) general investment in personal health. Some women seemed to have difficulty understanding prevention, and many felt greater clarity about microbicide use for treatment. Many viewed barriers (i.e., leakage, remembering, inserting) as easy to manage since they already used panty-liners, thought product use could be incorporated into daily routines and thought partners could help. Treating an active infection trumped barriers; this was less clear for prevention use. The decision regarding product use was viewed as belonging to the woman; partners were perceived as supporting what is best for the baby.

Conclusions Women in a NYC pre-natal clinic enthusiastically support the use of microbicides for RTIs. In order for this enthusiasm to lead to adoption, women need to be well-informed about the RTI, its consequences and risk/benefit of use. As barriers are likely to be greater in the absence of a current infection, a greater understanding and emphasis of the need versus the risk will be required for prevention use.

\section{P3.363 INFLAMMATORY SOLUBLE IMMUNE MEDIATORS AND PATHOGENIC VAGINAL BACTERIA IMPACT E. COLI BACTERICIDAL ACTIVITY IN FEMALE GENITAL TRACT SECRETIONS}

doi:10.1136/sextrans-2013-051184.0816

'R Pellett Madan, ${ }^{2,3} \mathrm{C}$ S Dezzutti, ${ }^{2,3} \mathrm{~L}$ Rabe, ${ }^{2,3} \mathrm{~S}$ L Hillier, ${ }^{4} \mathrm{~J}$ Marrazzo, ${ }^{2,3}$ McGowan, 4,5B A Richardson, 'B C Herold, Microbicide Trials Network Biomedical Sciences Working Group, 004MTN Protocol Team. 'Albert Einstein College of Medicine, Bronx, NY, United States; ' University of Pittsburgh School of Medicine, Pittsburgh, PA, United States; ${ }^{3}$ Magee-Womens Research Institute, Pittsburgh, PA, United States; ${ }^{4}$ University of Washington, Seattle, WA, United States; ${ }^{5}$ Fred Hutchinson Cancer Research Center, Seattle, WA, United States 